Rev. Elev. Méd. vét. Pays trop., 1973, 26 (1) : 77-80

\title{
Le Phacochère, Phacochærus athiopicus (Pallas, 1766), nouvel hôte pour Simondsia paradoxa Cobbold, 1864 (Nematoda; Spirurida) en Afrique tropicale
}

\author{
par J. BUSSIERAS (*)
}

\begin{abstract}
RESUME
L'auteur signale la présence de Simondsia paradoxa Cobbold, 1864, dans l'estomac d'un phacochère tué au Mali.

Il semble s'agir là, pour ce parasite, d'un hôte nouveau, et de la première observation en Afrique tropicale.
\end{abstract}

Simondsia paradoxa Cobbold, 1864 (Spiruridae; Ascaropsinae) est un Nématode parasite de l'estomac du porc domestique (Sus scrofa domestica L.) et du sanglier (Sus scrofa L.).

Il a été trouvé dans divers pays d'Europe et d'Asie. En Afrique, il n'a été signalé, à notre connaissance, que dans le nord du Maroc, chez des porcs domestiques abattus à Tétouan et à Melilla (6).

Il semble n'avoir jamais été rencontré en Afrique tropicale, ni chez le porc domestique (7), ni chez les Suidae sauvages (10).

Or, chez un phacochère, Phacocherus aethio picus (Pallas, 1766), tué sur les bords de la Falémé (environs de Bétakili, République du Mali), à proximité de la frontière sénégalaise, ont été trouvées de multiples lésions gastriques nodulaires, qui se révélèrent dues à la présence de Simondsia $(* *)$.

(*) Service de Parasitologie, Institut des Sciences et Médecine Vétérinaires de l'Université de Dakar (Sénégal).

(**) Nous remercions bien sincèrement M. le Professeur ALHINC, de la Faculté des Lettres de Dakar, qui a pris soin de prélever ces lésions et de nous les faire parvenir.

\section{DESCRIPTION SOMMAIRE DES PARASITES}

\subsection{Mâles}

Les mâles récoltés ont été trouvés partiellement enfoncés dans la muqueuse gastrique; ils étaient souvent disposés "en séton ", avec les deux extrémités libres dans la lumière.

Longueur : 8 à $12 \mathrm{~mm}$.

Diamètre maximal : $0,4 \mathrm{~mm}$.

Extrémité antérieure (fig. 1): orifice buccal limité par deux pseudo-lèvres latérales trilobées; à chaque lobe correspond une papille, très faiblement visible. Les deux pseudo-lèvres se réunissent dorsalement et ventralement en deux formations coniques proéminentes.

La bouche est suivie d'un vestibule buccal très allongé, dont la paroi présente des épaississements spiralés souvent peu marqués.

Latéralement, on observe une paire de papilles cervicales, suivie d'une paire d'ailes cervicales très développées. 


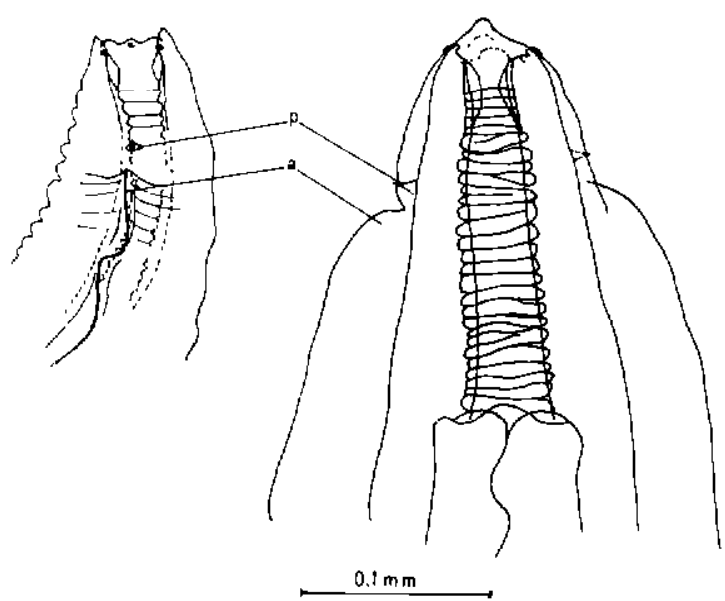

Fig. 1. - Simondsia paradoxa, extrémité antérieure; à gauche, mâle, vu latéralement; à droite, femelle, vue de face; a, aile cervicale; $p$, papille cervicale.

Extrémité postérieure (fig. 2 et 3 ): fortement spiralée, et portant ventralement de nombreuses verrucosités disposées en files longitudinales.

Les deux spicules sont très inégaux :

- spicule gauche 0,80 à $1,08 \mathrm{~mm}$

- spicule droit 0,35 à $0,42 \mathrm{~mm}$.

L'extrémité distale du spicule droit présente une petite expansion membraneuse.

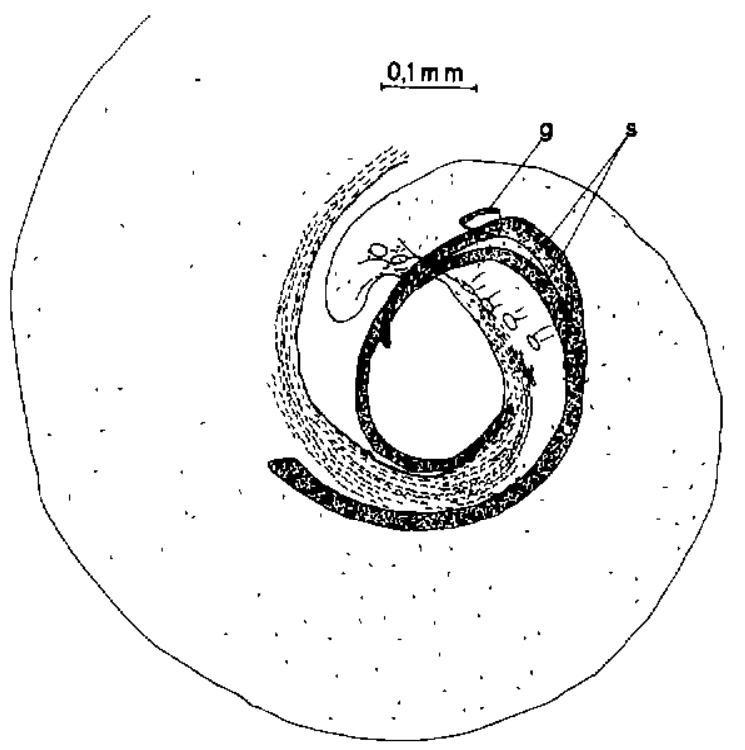

Fig. 2. - Simondsia paradoxa, mâle, extrémité postérieure vus latéralement; g, gubernaculum; s, spicules.

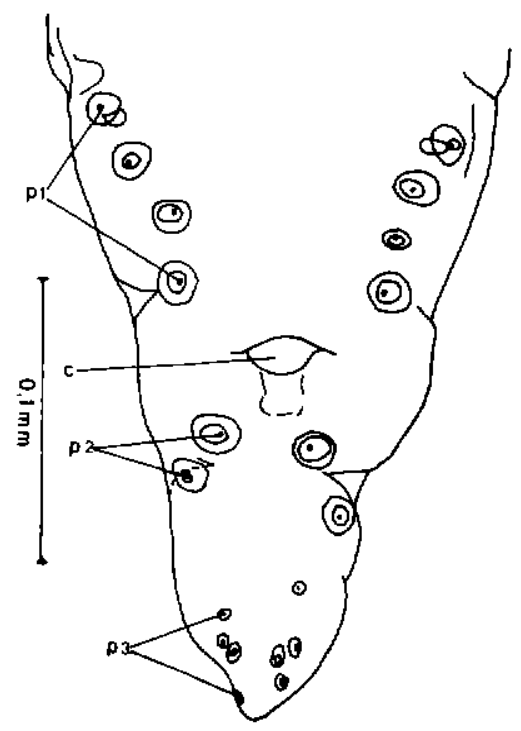

Fig. 3. - Simondsia paradoxa, mâle, extrémité postérieure vue de face; c, orifice cloacal; $\mathrm{p} 1$, papilles précloacales; $\mathbf{p} 2$, papilles postcloacales; $\mathbf{p} 3$, petites papilles terminales.

On observe un gubernaculum (longueur 36 à $57 \mu$ ), qui semble étroitement associé au spicule droit.

Latéralement, les deux ailes caudales sont associées à des papilles:

- 4 paires de papilles précloacales

- 2 paires de papilles postcloacales

- 4 paires de petites papilles terminales, très peu visibles, et à disposition asymétrique.

\subsection{Femelles}

Toutes les femelles récoltées étaient à maturité, avec la partie postérieure du corps globuleuse, située dans la sous-muqueuse gastrique, et la partie antérieure filiforme émergeant à la surface de la muqueuse. L'état de conservation des prélèvements n'a malheureusement pas permis l'extraction d'une femelle intacte.

Partie antérieure :

- longueur : 4 à $5 \mathrm{~mm}$

- diamètre : $0,1 \mathrm{~mm}$ vers le début de l'œsophage, mais augmentant progressivement pour atteindre 0,4 à $0,5 \mathrm{~mm}$.

Partie postérieure : diamètre 2 à $3 \mathrm{~mm}$.

L'extrémité antérieure est comparable à celle des mâles, mais les épaississements de la paroi du vestibule buccal sont beaucoup plus mar- 
qués. Très souvent, le ver est recourbé en crosse un peu en arrière du vestibule buccal.

\subsection{Cufs}

Les oufs, contenus dans les femelles, sont embryonnés; leur coque est à côtés parallèles; dimensions : $28-30 \times 11 \mu$.

\section{DISCUSSION}

Etant donné que ces parasites ont été récoltés dans une zone extrêmement peu accessible, où semble n'exister aucun élevage de porcs domestiques, il paraît très probable que l'on a affaire à des nématodes autochtones, propres au phacochère.

La question se pose donc de savoir s'il s'agit d'une espèce nouvelle, ou au contraire du même Simondsia paradoxa que chez le porc domestique et le sanglier d'Europe et d'Asie.

Malheureusement, l'espèce Simondsia paradoxa, caractérisée notamment par la morphologie de l'extrémité postérieure du mâle, a fait l'objet de descriptions nombreuses et plus ou moins discordantes.

C'est ainsi que, pour les papilles génitales du mâle, on trouve les renseignements suivants :

\begin{tabular}{|l|c|c|c|}
\hline & $\begin{array}{c}\text { Papilles } \\
\text { précloacales }\end{array}$ & $\begin{array}{c}\text { Papilles } \\
\text { postcloacales }\end{array}$ & $\begin{array}{c}\text { Petites papilles } \\
\text { terminales }\end{array}$ \\
\hline PIANA, 1897 & 3 à 4 paires & 1 à 2 paires & 0 \\
SIMON, 1950 & 4 paires & 1 paire & 4 paires \\
LOPEZ-NEYRA, 1951 & 4 paires & 2 paires & 4 à 5 paires \\
RAMANUJACHARI et ALWAR, 1953 & 4 paires & 1 paire & 2 à 3 paires \\
KOIAVA, 1954 & 5 paires & 1 paire & 3 paires \\
AHLUWALIA, 1962 & 4 paires & 2 paires & 2 papilles médianes \\
GUPTA et SOOD, 1968 & 5 parres & 2 paires & 3 papilles médianes \\
\hline
\end{tabular}

KOIAVA avait étudié des Simondsia récoltés sur des sangliers en Géorgie soviétique, et avait considéré qu'il s'agissait d'une espèce nouvelle, Simondsia petrowi, espèce ultérieurement admise par YAMAGUTI (12). Cependant, DIMITROVA (3) a montré que ces vers étaient identiques aux $S$. paradoxa des porcs et sangliers de Bulgarie, et que $S$. petrowi devait donc tomber en synonymie.

Nous admettrons que toutes ces divergences sont dues aux difficultés d'observation, notamment en ce qui concerne les petites papilles terminales, et au fait que la spirale très marquée de l'extrémité postérieure du mâle rend délicate l'obtention de vues de face de cette portion (fig. 3).
Par ailleurs, il serait nécessaire de reprendre 1'étude de Simondsia cesticillus (Molin, 1859), parasite de l'estomac d'un pécari sud-américain, Dicotyles albirostris ( $=$ Tayassu pecari), car LOPEZ-NEYRA (6), suivi par AHLUWALIA (1), considèrent que $S$. paradoxa de l'ancien monde n'est qu'une forme pathologique (en ce qui concerne les femelles) de la même espèce. L'« anomalie » représentée par l'hypertrophie des femelles serait liée à une adaptation incomplète du parasite au porc et au sanglier. Il est intéressant de remarquer que le phacochère africain, zoologiquement bien différent des Suidae européens, héberge cependant des $\mathrm{Si}$ mondsia dont les femelles sont également hypertrophiées.

\section{SUMMARY}

The warthog, Phacochoerus aethiopicus (Pallas, 1766), as new host of Simondsia paradoxa Cobbold, 1864 (Nematoda; Spiruridae) in tropical Africa

The author brings to notice the presence of Simondsia paradoxa Cobbold, 1864, in the stomach of a warthog killed in Mali.

It seems it $1 \mathrm{~s}$ the first observation of that parasite in tropical Africa and in a warthog as host. 


\section{RESUMEN}

El facoquerio, Phacochoerus aethiopicus (Pallas, 1766), nuevo huesped para Simondsia paradoxa Cobbold, 1864 (Nematoda; Spiruridae) en Africa tropical

El autor señala la presencia de Simondsia paradoxa Cobbold, 1864, en el estómago de un facoquerio matado en Mali.

Parece que se trata, en lo concerniente a dicho parásito, de un huesped nuevo y de la primera observación en Africa tropical.

\section{BIBLIOGRAPHIE}

1. AHLUWALIA (S.S.). Simondsia paradoxa Cobbold, a redescription with remarks on the hostparasite relationship. Indian J. vet. Sci. anim. Husb., 1960, 30 (3) : 191-199.

2. AHLUWALIA (S. S.). Studies on some helminths of the domestic pig (Sus scrofa domestica) in western Uttar Pradesh. J. Helminth., 1962, 36 (4) : 347-364.

3. DIMTTROVA (E.). Révision des membres du genre Simondsia. Izv. tsent. khelmint. Lab., Sofia, 1962, $7: 43-50$.

4. GUPTA (N. K.), SOOD (M. L.). Observations on four already known nematodes of pigs from the Punjab. Res. Bull. Panjab Univ. Sci., 1968, 19 (3-4) : 277-290.

5. KOLAVA (L. I.). Une nouvelle espèce de nématode - Simondsia petrowi $\mathrm{n}$. sp. -- du sanglier de Géorgie. Soobsh. Akad. Gruzinsk. SSR, 1954, $15(7): 467-472$.

6. LOPEZ NEYRA (C. R.). Los Ascaropsinae (Nematoda - Spirurata). Revta ibér. Parasit., 1951, 11 (2): $89-223$.
7. MOREL (P. C.). Les helminthes des animaux domestiques de l'Afrique occidentale - Revue. Rev. Elev. Méd. vét. Pays trop., 1959, 12 (2): 153-174.

8. PIANA (G. P.). Ricerche sulla morfologia della Simondsia paradoxa Cobbold. Atit Soc. ital. Sci. nat., Milan, 1897, 37 (1): 17-37.

9. RAMANUJACHARI (G.), ALWAR (V.S.). On Simondsia paradoxa Cobbold 1864 - a parasitic helminth of pigs. Indian vet. J., 1953, 29 (5) : 381382.

10. ROUND (M. C.). Check list of the helminth parasites of African mammals. Farnham Royal, Bucks, Commonwealth Agricultural Bureaux, 1968.

11. SIMON VICENTE (F.). Nematodos parásiticos gástricos del Sus scrofa domesticus L. de España, Revta ibér. Parasit., 1950, 10 (3) : 363-375.

12. YAMAGUTI (S.). Systema helminthum. Vol. III. The nematodes of vertebrates. New York, Interscience Publishers, 1961. 